\title{
Identification of Plastic Wastes by Using Fuzzy Radial Basis Function Neural Networks Classifier with Conditional Fuzzy C-Means Clustering
}

\author{
Seok-Beom Roh* and Sung-Kwun $\mathrm{Oh}^{\dagger}$
}

\begin{abstract}
The techniques to recycle and reuse plastics attract public attention. These public attraction and needs result in improving the recycling technique. However, the identification technique for black plastic wastes still have big problem that the spectrum extracted from near infrared radiation spectroscopy is not clear and is contaminated by noise. To overcome this problem, we apply Raman spectroscopy to extract a clear spectrum of plastic material. In addition, to improve the classification ability of fuzzy Radial Basis Function Neural Networks, we apply supervised learning based clustering method instead of unsupervised clustering method. The conditional fuzzy C-Means clustering method, which is a kind of supervised learning based clustering algorithms, is used to determine the location of radial basis functions. The conditional fuzzy C-Means clustering analyzes the data distribution over input space under the supervision of auxiliary information. The auxiliary information is defined by using k Nearest Neighbor approach.
\end{abstract}

Keywords: Identification of plastic wastes, Conditional fuzzy C-means clustering, k nearest neighbor approach, Fuzzy radial basis function neural networks

\section{Introduction}

These days, one key issue of mankind is the alteration of global weather patterns. The alteration of weather patterns is assumed to be caused by global warming. Many industrialized countries are trying to slow down global warming by reducing carbon dioxide emission. One way to reduce carbon dioxide emission as well as the pace of energy depletion is the waste recycling and reuse.

Among the various municipal wastes generated every day around world, plastic is one of major material. It is natural that the techniques to recycle and to reuse plastic waste come into the spotlight. The various techniques to recycle and reuse plastic waste have been studied. The key issue in recycling and reusing plastic waste is the identification plastic material. When recycling and reusing plastic waste, the purity of the recycled plastic is very important. To obtain homogeneous plastic material, the identification technique is need.

In 1995, Scott proposed an identification technique to identify polyethylene terephthalate (PET) and polyvinyl chloride (PVC) resins [1]. The identification technique proposed by Scott was based on Near Infrared Radiation (NIR) to extract absorbance spectra of each plastic material. In addition, a method to classify plastic resins into PET and PVC categories was proposed by Edward and Sommer in 2001 [2]. The classification system invented by Edward Sommer was based on NIR transmission spectra of

$\dagger$ Corresponding Author: Dept. of Electrical Engineering, The University of Suwon, Korea. (ohsk@suwon.ac.kr)

* Dept. of Electrical Engineering, The University of Suwon, Korea. (rsb@suwon.ac.kr)

Received: March 7, 2016; Accepted: June 13, 2016 each material. NIR spectroscopy equipment is applicable to classify and identify plastic material whose color is not black. However, when classifying and identifying black plastic which contains carbon black material, NIR spectroscopy equipment is not able to be used for a sensor. Because black colored materials can absorb light of all wave length. In this paper, to overcome the drawback of NIR spectroscopy, Raman spectroscopy technique is used. Nowadays, Raman spectroscopy technique is used in very wide applications such as pharmaceuticals, forensics, archeology, art, medicine, semiconductors in microelectronics, geology, planetary science and materials science [3]. It is well known that Raman spectroscopy is a spectroscopic technique exploiting the inelastic scattering phenomenon of monochromatic light, usually from a laser source [4]. Inelastic scattering means that the frequency of photons in monochromatic light from a light source changes by interacting with a material.

In addition, in order to improve identification performance of the identification system to classify some plastics into several categories, intelligent techniques such as fuzzy set theory and neural networks are used. Especially, fuzzy Radial Basis Function Neural Networks (fRBFNNs) are used as a classifier to identify black plastic wastes. In general, it is well known that these intelligent techniques have same advantages of dealing with the uncertain data and having learning ability. The locations of radial basis functions are very important to improve the classification performance of fRBFNNs. The locations of radial basis function are determined by using unsupervised clustering technique (usually fuzzy C-means clustering method). However, the unsupervised clustering method does not use 
the auxiliary information that can be extracted the output space but the data distribution information extracted over the input space [5]. We use conditional fuzzy C-means clustering (cFCM) algorithm to define radial basis functions in supervised learning manner. The auxiliary information over the output space is defined by fuzzy kNearest Neighbors (kNN) approach.

In this paper, we make some experiments with several machine learning standard datasets to verify the proposed intelligent identification system. Finally, we use the proposed identification algorithm to classify black plastics into several material categories.

\section{Fuzzy C-Means Clustering and Conditional Fuzzy C-Means Clustering}

The location of radial basis functions is usually defined by using unsupervised method that analyzes the data distribution on the input space. In some literatures, this step to analyze the data distribution can be considered as the granulation of information [6-8]. The information granules are extracted by analyzing numerical data distribution and other source of experimental evidence. In this study, we use here Fuzzy C-Means clustering algorithm to extract fuzzy set.

\subsection{Fuzzy C-means clustering algorithms}

A brief explanation of the fuzzy clustering algorithm is outlined below.

Let us consider a finite set of data $\mathbf{X}=\left\{\mathbf{x}_{1}, \mathbf{x}_{2}, \cdots, \mathbf{x}_{N}\right\}$, $\mathbf{x}_{k} \in \mathfrak{R}^{m}, 1 \leq k \leq N$. The FCM clustering should optimize the following objective function.

$$
J_{p}=\sum_{i=1}^{c} \sum_{k=1}^{N}\left(w_{i k}\right)^{p}\left\|\mathbf{x}_{k}-\mathbf{v}_{i}\right\|^{2}, \quad 1<p<\infty
$$

The above optimization is carried out subject to the following constraints.

$$
\begin{gathered}
\sum_{i=1}^{c} w_{i k}=1 \\
0<\sum_{k=1}^{N} w_{i k}<N
\end{gathered}
$$

Here, $\left\|\mathbf{x}_{k}-\mathbf{v}_{i}\right\|$ is any distance between the data $\mathbf{x}_{k}$ and the prototype $\mathbf{v}_{i}$. The parameter " $p$ " used in the objective function denotes the fuzzification coefficient. " $N$ " and " $c$ " mean the number of data patterns and clusters, respectively. Usually, the fuzzification coefficient is equal to 2 . The distance between data and prototypes is considered as the generalized weighted version of Euclidean distance as (3).

$$
\|\mathbf{a}-\mathbf{b}\|^{2}=\sum_{l=1}^{m} \frac{\left(a_{l}-b_{l}\right)^{2}}{\sigma_{l}^{2}}
$$

Here, $\sigma_{l}^{2}$ is the variance of the $l$-th variable, $l=1,2, \ldots, \mathrm{m}$. In fuzzy clustering procedure, the objective function is minimized by sequential optimization operation which optimizes the partition matrix and the associated prototypes.

Partition matrix $\boldsymbol{W}: w_{i k}$ is the i-th row, $\mathrm{j}$-th column element of partition matrix $\boldsymbol{W}$

$$
w_{\mathrm{ik}}=\frac{1}{\sum_{l=l}^{c}\left(\frac{\left\|\mathbf{x}_{\mathrm{k}}-\mathbf{v}_{\mathrm{i}}\right\|}{\left\|\mathbf{x}_{\mathrm{k}}-\mathbf{v}_{l}\right\|}\right)^{2 /(p-1)}}
$$

Prototypes $\boldsymbol{v}_{1}, \boldsymbol{v}_{2}, \ldots, \boldsymbol{v}_{\mathrm{c}}$

$$
\mathbf{v}_{i}=\frac{\sum_{k=1}^{N} w_{i k}^{p} \mathbf{x}_{k}}{\sum_{k=1}^{N} w_{i k}^{p}}
$$

The partition matrix and the prototypes are updated in an iterative fashion and the iterative optimization process keeps proceeding until an already determined certain termination criterion has been satisfied.

\subsection{Conditional fuzzy c-means clustering algorithms}

In order to determine the center points of radial basis functions of fRBFNNs, unsupervised clustering algorithms are generally used. As mentioned before, conventional unsupervised clustering methods cannot use the whole information involved in the given data patterns. Conventional FCM algorithm is known as a sort of unsupervised learning algorithms. The supervised learning version of conventional FCM is conditional fuzzy C-means clustering algorithm (cFCM) that was proposed by Pedrycz [9]. cFCM can analyze input space under supervision of other auxiliary information that can be defined by a designer or from the experimental evidence.

In this study, we apply cFCM to define the center points of radial basis functions. When applying $\mathrm{cFCM}$ to analyze the data distribution, the input data pattern $\mathbf{x}_{k} \in \mathfrak{R}^{m}$ are grouped by taking into account the auxiliary information $\left(f_{1}, f_{2}, \cdots, f_{N}\right)$. While the objective function for cFCM is the same as (1), the constraints are different from (2). The constraint for $\mathrm{cFCM}$ is as follows.

$$
\sum_{i=1}^{c} w_{i k}=f_{k}
$$

The optimization problem to minimize (1) subject to a constraint (6) is expressed as (7). 


$$
\min _{\mathbf{w}, \mathbf{v}} J \text { subject to } \sum_{i=1}^{c} w_{i k}=f_{k}
$$

The optimal solution that minimizes the objective function (1) subject to (6) can be obtained by iterative update formulas such as (8) and (9).

$$
\begin{gathered}
w_{i k}=\frac{f_{k}}{\sum_{j=1}^{C}\left(\frac{\left\|\mathbf{x}_{k}-\mathbf{v}_{i}\right\|}{\left\|\mathbf{x}_{k}-\mathbf{v}_{j}\right\|}\right)^{2 /(p-1)}} \\
\mathbf{v}_{i}=\frac{\sum_{\mathrm{k}=1}^{\mathrm{N}}\left(u_{i k}\right)^{p} \cdot \mathbf{x}_{k}}{\sum_{\mathrm{k}=1}^{\mathrm{N}}\left(u_{i k}\right)^{p}}
\end{gathered}
$$

\section{3 k-Nearest neighbor approach for auxiliary information}

To apply the above explained cFCM, the already defined auxiliary information should be needed. We use $\mathrm{k}$ Nearest Neighbor approach to extract the auxiliary information which is defined on the output space. We can say that the data point, whose nearest neighbors are involved in the same class, has homogeneous characteristic. The heterogeneous data point is defined as the data point whose nearest neighbors are involved in different classes.

In order to build homogeneous clusters over the input space, the homogeneous data points should be considered more importantly in comparison with the heterogeneous data points. Therefore, we define the auxiliary information of a given data point as how much homogeneous the data point is. In pattern recognition fields, $\mathrm{kNN}$ approach is considered as a kind of non-parametric method to directly classify the given data point. On the contrary, we use kNN approach to extract the auxiliary information.

We define the auxiliary information as (10).

$$
f_{i j}=\frac{n_{i j}}{K}
$$

Here, $f_{i j}$ denotes the auxiliary information of the $\mathrm{j}$-th data point $\mathbf{x}_{j}$ on the i-th class, $\mathrm{K}$ is the number of the nearest neighbors of the given data point, and $n_{i j}$ means the number of the nearest neighbors of the given data which are involved in i-th class. The auxiliary information defined by (10) should satisfy (11).

$$
\sum_{j=1}^{L} f_{i j}=\sum_{j=1}^{L} \frac{n_{i j}}{K}=1
$$

Here, L means the number of classes.

\section{Radial Basis Function Neural Networks based on Auxiliary Information}

On the contrary to the general definition methods for the locations of RBFs which are based on the unsupervised clustering algorithm, we use cFCM to determine the locations of RBFs under the supervision of the auxiliary information. The auxiliary information includes the information of the class label of the given data point. This statement means that we can use the information, which the given data patterns represent over input space an output space, as much as we can use.

In some literatures $[10,11]$, it is said that the RBFNNs have some advantages including global optimal approximation and classification capabilities as well as rapid convergence of the underlying learning procedures.

The generic topology of RBF NNs is depicted in Fig. 1. In Fig. 1, $\Gamma_{i}, \mathrm{i}=1,2, \ldots, \mathrm{c}$ denote receptive fields (radial basis functions), while the parameter " $\mathrm{m}$ " denotes the number of input variables. The output of the generic RBF $\mathrm{NN}$ is expressed as a linear combination of the outputs $(\Gamma(\mathbf{x}))$ of the corresponding hidden nodes with the connection weights $w_{1}, w_{2}, \cdots, w_{c}$ as (12).

$$
\hat{y}(\mathbf{x})=\sum_{i=1}^{c} w_{i} \cdot \Gamma_{i}(\mathbf{x})
$$

Here, $\Gamma(\mathbf{x})$ means the activation level of $i$-th hidden node.

We expand the generic RBFNNs by adopting cFCM to locate RBFs with the aids of auxiliary information and applying polynomials to connection weights. In the expanded version of RBFNNs, the auxiliary information extracted by $\mathrm{kNN}$ help cFCM determine the location of RBFs in the viewpoint of the homogeneity of the data points involved in the RBFs. We assume that in order to improve the identification performance, the data points which are involved in a cluster defined by a RBF should be homogeneous. To obtain the homogeneous clusters, we

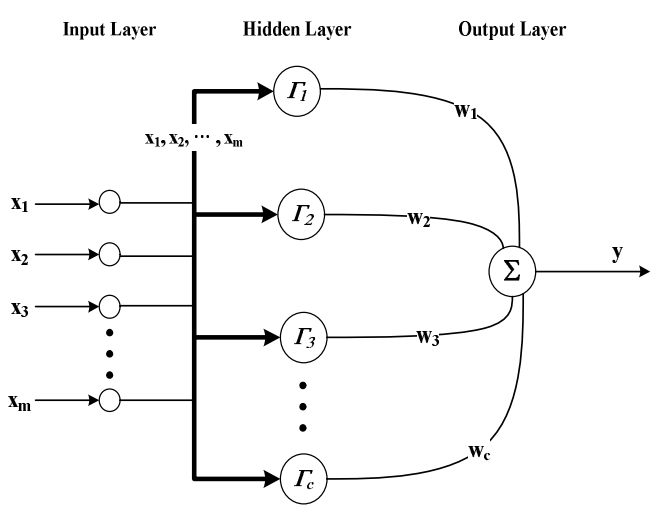

Fig. 1. General architecture of the generic RBF neural networks 


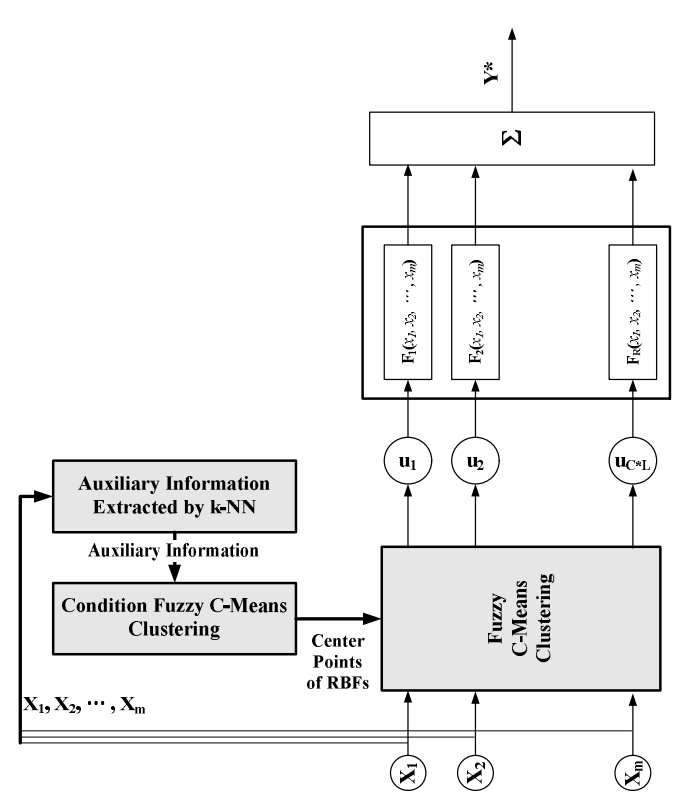

Fig. 2. An overall topology of radial basis function neural networks with auxiliary information

apply cFCM with the auxiliary information obtained by using $\mathrm{kNN}$. The auxiliary information through $\mathrm{kNN}$ means how much homogeneous the data points are.

The overall topology of the Radial Basis Function Neural Networks with the auxiliary information is shown in Fig. 2.

We use three types of polynomials as the consequent functions of the proposed RBF neural networks as follows.

$$
\begin{gathered}
F_{j}=a_{j} \\
F_{j}=a_{j 0}+a_{j 1} x_{1}+\cdots+a_{j m} x_{m} \\
F_{j}=a_{j 0}+\sum_{k=1}^{m} a_{j k} x_{k}+\sum_{k=1}^{m} a_{j, m+k} x_{k}^{2}
\end{gathered}
$$

The output of RBF neural networks is defined as (14).

$$
\hat{y}\left(\mathbf{x}_{k}\right)=\frac{\sum_{i=1}^{c \times L} w_{i k} f_{i}\left(\mathbf{x}_{k}\right)}{\sum_{i=1}^{c \times L} w_{i k}}
$$

\section{Extraction of Input Variables from Raman Spectrum}

For implementing the identification system of black plastic, Raman spectroscopy is used as a sensor to obtain the specific data pattern from a black plastic material.

Generally, Raman spectroscopy investigates materials not through direct absorption, but by scattering of high intensity light in the hopes that one in a million photons

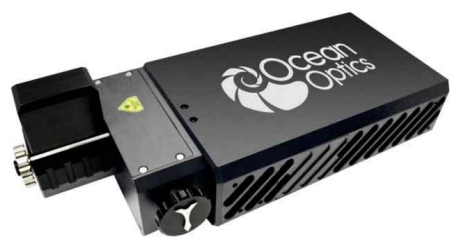

Fig. 3. Integrated raman spectrometer produced by ocean optics

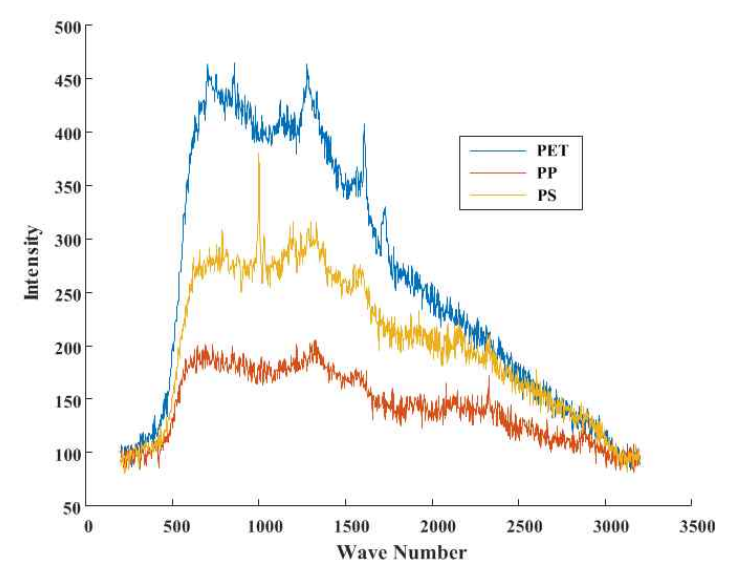

Fig. 4. Spectra of plastics such as PET, PP, and PS obtained from RAMAN spectrometer

scattered will commune with the vibrational and rotational states of a sample molecule and emit light of a slightly different wavelength.

The Raman spectroscopy equipment used in this experiment is produced by Ocean Optics (www.oceanoptics.com). The Raman equipment is shown in Fig. 3.

The light source of the Raman spectroscopy equipment is $785 \mathrm{~nm}$ diode laser. The spectrum acquired from the above mentioned Raman spectroscopy of each plastic material is shown in Fig. 4. The raw spectrum obtained from Raman spectrometer should be preprocessed to be used as the input of the intelligent classifier (in this study, fRBFNNs).

We should determine at which wave number the characteristic peaks happens according plastic materials such as PET, PP, and PS. The information of characteristic peaks of each plastic material can be obtained from the field experts. In this study, we do not try to depend on the field experts' knowledge but to extract the information from the raw spectra obtained from Raman spectroscopy.

In order to distinguish a plastic material from the other plastic materials, we extract unique wave number where the unique peaks of a specific plastic material happen. We compare a spectrum of a specific plastic material with the spectra of the other plastic materials. In this comparison operation, the "XOR" operator is used and is defined as (15).

$$
X O R(\mathbf{S}, \mathbf{T})=(1-\mathbf{S}) \otimes \mathbf{T}+\mathbf{S} \otimes(1-\mathbf{T})
$$


Here, $\otimes$ means the element-wise product.

For example, let the spectrum $\mathrm{A}$ and $\mathrm{B}$ be $\mathbf{A}=\left[\begin{array}{llll}a_{1} & a_{2} & \cdots & a_{q}\end{array}\right]^{T}$ and $\mathbf{B}=\left[\begin{array}{llll}b_{1} & b_{2} & \cdots & b_{q}\end{array}\right]^{T}$ respectively.

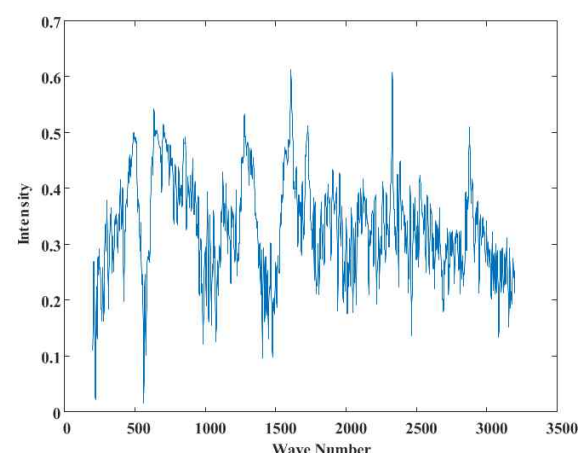

(a) XOR operation between PET and PP

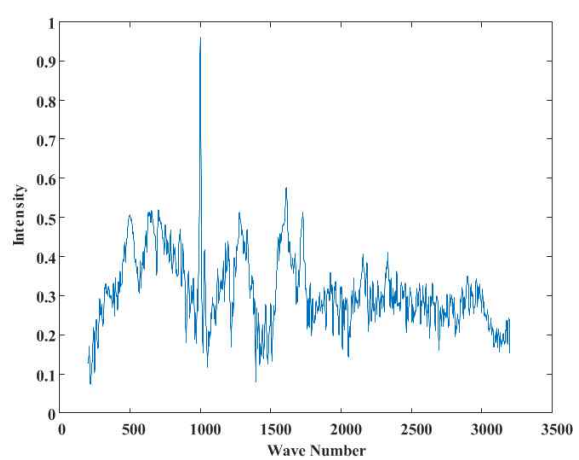

(b) XOR operation between PET and PS

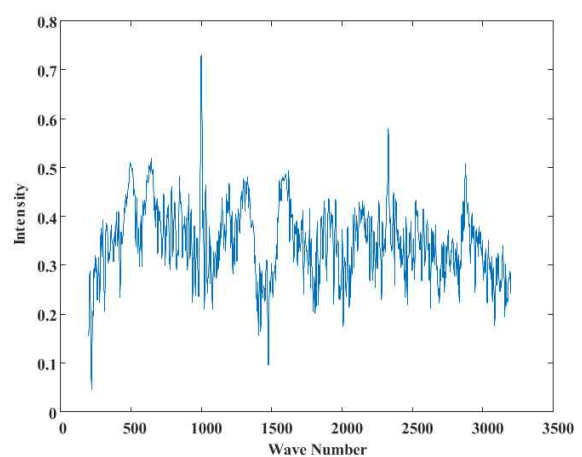

(c) XOR operation between PP and PS

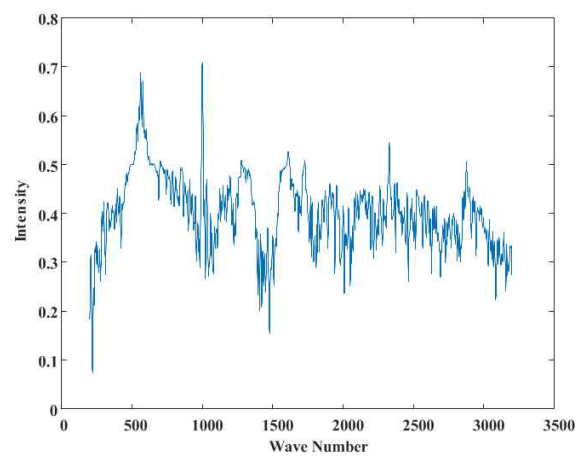

(d) XOR operation between three types of plastic materials

Fig. 5. XOR operation result between plastic
The result of " $\otimes$ " operation of two spectra is calculated as $\mathbf{A} \otimes \mathbf{B}=\left[\begin{array}{lllll}a_{1} \cdot b_{1} & a_{2} \cdot b_{2} & \cdots & a_{q} \cdot b_{q}\end{array}\right]^{T}$.

The results of XOR operation between two different plastic materials are shown in Fig. 5.

The element-wise operation version of (15) is $\operatorname{xor}(a, b)=(1-a) \cdot b+a \cdot(1-b)$.

In the case of $a=0 \& b=1$ or $a=1 \& b=0$, the output of the operation is maximized. In other words, when the difference between two spectra is big, the output of the XOR operation becomes big. We can consider the 15 maximum points of the result of the "XOR" operation between three different plastic materials as the characteristic peaks which are shown in only one plastic material.

In the plastic material identification experiment, we obtain 100 raw spectra for each plastic material and total 300 row spectra (100 PET, 100 PP, and 100 PS). From this "XOR" operation, we can estimate 15 characteristic peaks as shown in Table 1.

\section{Experimental Studies}

We make several experiments in order to verify the proposed design method based on $\mathrm{k}-\mathrm{NN}$ and C-FCM to implement RBF neural networks. In these experiments, we use a series of machine learning datasets (http://www. ics.uci.edu/ mlearn/MLRepository.html).

In this experiment, we use classification rate to evaluate the proposed classifier and 10-Fold cross-validation to evaluate the classification result.

In addition, to verify the black plastic identification performance of the proposed intelligent classifier, we use black plastic raw spectra from Raman spectroscopy equipment. At first, we should predefine some design parameters whose values are summarized in Table 2.

Table 3 summarizes the pertinent details of each dataset

Table 1. The characteristic peaks estimated by "XOR" operation

\begin{tabular}{c|c|c|c|c|c}
\hline No. & $\begin{array}{c}\text { Wave } \\
\text { Number }\end{array}$ & No & $\begin{array}{c}\text { Wave } \\
\text { Number }\end{array}$ & No & $\begin{array}{c}\text { Wave } \\
\text { Number }\end{array}$ \\
\hline 1 & 493 & 6 & 853 & 11 & 1612 \\
\hline 2 & 640 & 7 & 999 & 12 & 1656 \\
\hline 3 & 706 & 8 & 1282 & 13 & 1725 \\
\hline 4 & 724 & 9 & 1367 & 14 & 2330 \\
\hline 5 & 746 & 10 & 1553 & 15 & 2876 \\
\hline
\end{tabular}

Table 2. The selected numerical values of the parameters of the proposed classification technique

\begin{tabular}{c|c}
\hline Parameter & Value \\
\hline Number of Rules in each class $(\mathrm{C})$ & $2,3,4,5$ \\
\hline Number of Nearest Neighbors $(\mathrm{K})$ & $3,5,7,10,20,30$ \\
\hline Fuzzification Coefficient $(\mathrm{p})$ & $1.2,1.5,2.0,2.5,3.0$ \\
\hline Polynomial Type $(\mathrm{T})$ & $1,2,3$ \\
\hline
\end{tabular}


Table 3. Machine learning datasets used in the experiments

\begin{tabular}{c|c|c|c}
\hline Data set & $\begin{array}{c}\text { Number } \\
\text { of Classes }\end{array}$ & $\begin{array}{c}\text { Number } \\
\text { of features }\end{array}$ & $\begin{array}{c}\text { Number } \\
\text { of patterns }\end{array}$ \\
\hline Australian & 2 & 42 & 690 \\
Balance & 3 & 4 & 625 \\
Bupa & 2 & 6 & 347 \\
German & 2 & 24 & 1000 \\
Glass & 6 & 9 & 214 \\
Hayes & 3 & 5 & 132 \\
Ionosphere & 2 & 34 & 351 \\
Iris & 3 & 4 & 150 \\
Pima & 2 & 8 & 768 \\
Sonar & 2 & 60 & 208 \\
Thyroid & 3 & 5 & 215 \\
Vehicle & 4 & 18 & 846 \\
Wine & 3 & 13 & 178 \\
Zoo & 7 & 16 & 101 \\
\hline
\end{tabular}

Table 4. Classification performance of the proposed classifier and generic RBF neural networks

\begin{tabular}{c|c|c|c|c|c|c|c|c}
\hline \multirow{2}{*}{ Data Sets } & \multicolumn{4}{|c|}{ Generic RBF NN } & \multicolumn{4}{c}{ Proposed RBF NN } \\
\cline { 2 - 9 } & Training Data & \multicolumn{2}{c|}{ Test Data } & \multicolumn{2}{c}{ Training Data } & \multicolumn{2}{c}{ Test Data } \\
\cline { 2 - 9 } & mean & STD & mean & STD & mean & STD & mean & STD \\
\hline Australian & 84.36 & 1.24 & 85.65 & 4.61 & 86.23 & 0.81 & $\mathbf{8 6 . 3 8}$ & 4.33 \\
\hline Balance & 89.17 & 0.94 & $\mathbf{8 8 . 1 6}$ & 3.72 & 79.11 & 0.63 & 78.87 & 4.91 \\
\hline Bupa & 59.91 & 2.67 & 57.39 & 9.49 & 67.34 & 1.01 & $\mathbf{6 2 . 9 5}$ & 7.27 \\
\hline German & 67.07 & 0.80 & 67.00 & 3.40 & 69.71 & 0.62 & $\mathbf{7 0 . 0 0}$ & 4.57 \\
\hline Glass & 66.82 & 1.88 & 60.76 & 11.70 & 65.99 & 0.98 & $\mathbf{6 2 . 6 2}$ & 8.22 \\
\hline Hayes & 54.21 & 4.15 & 53.19 & 10.26 & 74.24 & 3.47 & $\mathbf{6 6 . 8 7}$ & 10.95 \\
\hline Ionosphere & 91.50 & 1.13 & $\mathbf{8 9 . 8 4}$ & 4.79 & 90.63 & 1.16 & $\mathbf{9 0 . 9 8}$ & 4.87 \\
\hline Iris & 95.85 & 1.12 & 96.00 & 7.17 & 96.37 & 0.89 & $\mathbf{9 7 . 3 3}$ & 3.44 \\
\hline Pima & 69.78 & 1.28 & 70.05 & 4.14 & 74.65 & 0.61 & $\mathbf{7 3 . 6 9}$ & 3.64 \\
\hline Sonar & 75.75 & 1.20 & 75.00 & 7.50 & 84.83 & 1.46 & $\mathbf{8 1 . 7 1}$ & 7.21 \\
\hline Thyroid & 95.92 & 0.90 & $\mathbf{9 7 . 6 6}$ & 3.33 & 96.49 & 0.41 & $\mathbf{9 7 . 2 1}$ & 3.22 \\
\hline Vehicle & 59.26 & 1.38 & 57.68 & 3.92 & 69.33 & 0.75 & $\mathbf{6 7 . 1 3}$ & 4.47 \\
\hline Wine & 97.13 & 0.73 & $\mathbf{9 7 . 7 8}$ & 3.88 & 95.57 & 0.55 & $\mathbf{9 7 . 2 2}$ & 3.93 \\
\hline Zoo & 99.23 & 1.05 & $\mathbf{9 6 . 0 0}$ & 6.99 & 97.47 & 0.90 & 93.00 & 8.23 \\
\hline
\end{tabular}

Table 5. The identification performance of the proposed plastic identification system

\begin{tabular}{c|c|c|c|c|c|c|c|c|c|c|c}
\hline \multirow{2}{*}{$\mathrm{m}$} & \multirow{2}{*}{$\mathrm{R}$} & \multirow{2}{*}{$\mathrm{p}$} & \multirow{2}{*}{$\mathrm{K}$} & \multicolumn{3}{|c|}{$\mathrm{C}$} & \multicolumn{2}{c|}{ Training Data } & \multicolumn{2}{c}{ Test Data } \\
\cline { 5 - 12 } & & & & $\mathrm{C} 1$ & $\mathrm{C} 2$ & $\mathrm{C} 3$ & mean & STD & mean & STD \\
\hline 7 & 5 & 2.0 & 10 & 4 & 4 & 4 & 86.50 & 2.35 & 86.0 & 3.65 \\
\hline 7 & 7 & 2.0 & 3 & 4 & 3 & 4 & 87.75 & 1.20 & 87.0 & 2.17 \\
\hline 9 & 5 & 1.5 & 7 & 3 & 3 & 4 & 86.92 & 1.00 & 85.67 & 3.46 \\
\hline 9 & 7 & 1.5 & 7 & 3 & 4 & 4 & 86.75 & 2.07 & 84.67 & 2.17 \\
\hline 15 & 5 & 3.0 & 10 & 4 & 3 & 3 & 92.92 & 1.21 & 93.33 & 6.56 \\
\hline 15 & 7 & 3.0 & 7 & 2 & 3 & 4 & 93.25 & 0.62 & 93.33 & 3.53 \\
\hline 20 & 5 & 3.0 & 10 & 2 & 4 & 2 & 90.08 & 1.68 & 90.67 & 4.80 \\
\hline 20 & 7 & 3.0 & 3 & 3 & 3 & 3 & 90.83 & 1.77 & 91.0 & 4.35 \\
\hline
\end{tabular}

such as the number of classes, number of features, and number of patterns.

Table 4 summarizes the classification performance (\%) of the proposed neural networks and the generic RBF neural networks. From the experimental results shown in Table 4, the proposed RBFNNs are generally superior to the generic RBFNNs in the majority cases of data sets in terms of the prediction capability.

In Fig. 6, the spectra of plastics obtained from Raman

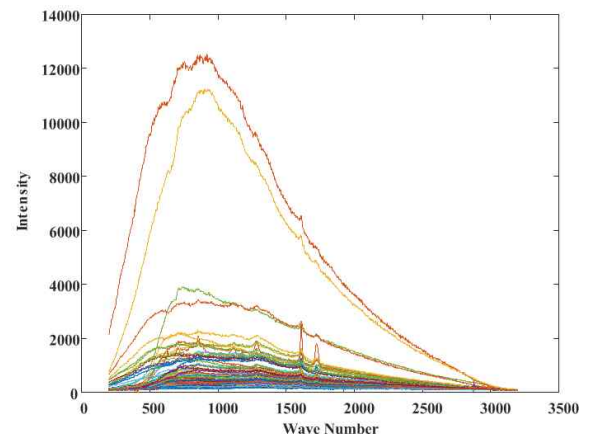

(a) PET

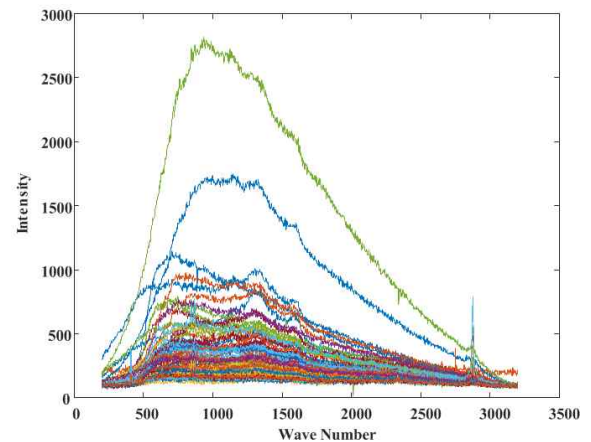

(b) PP

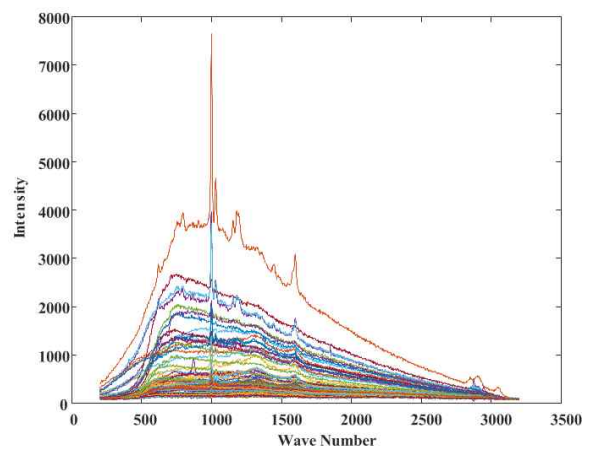

(C) PS

Fig. 6. Raman spectra of sampled plastics such (a) PET, (b) $\mathrm{PP}$, and (C) PS

spectroscopy such as PP, PS, and PET are shown.

In this black plastic identification experiment, the constant is used as the output polynomial used in fRBFNNs classifier. Because the higher order polynomial may result in the over-fitting problem.

Table 5 summarizes the identification performance (expressed \%) of the developed neural networks with respect to the number of selected variables $(\mathrm{m})$, the number of nearest neighbors $(\mathrm{K})$, the range of the input variables (R), the number of radial basis functions (C), and the fuzzification coefficient ( $\mathrm{p})$. The best result of the proposed identification system is the RBFNN with 9 RBFs, 3.0 fuzzification coefficient. The prediction performance of the best RBFNN is $93.33 \%$.

In order to validate the identification ability of the 
Table 6. Results of comparative analysis

\begin{tabular}{c|c|c|c}
\hline & Parameters & Training Data & Test Data \\
\hline $\begin{array}{c}\text { Proposed } \\
\text { Classifier }\end{array}$ & $\begin{array}{c}\mathrm{R}=7, \mathrm{~m}=15, \\
\mathrm{p}=3.0, \mathrm{~K}=7\end{array}$ & $93.25 \pm 0.62$ & $93.33 \pm 3.53$ \\
\hline $\begin{array}{c}\text { Bayes } \\
\text { Networks }\end{array}$ & $\mathrm{R}=5, \mathrm{~m}=7$ & $\mathrm{~N} / \mathrm{A}$ & 77.60 \\
\hline \multirow{2}{*}{$\mathrm{SVM}$} & $\begin{array}{c}\mathrm{R}=5, \mathrm{~m}=7 \\
(\text { Polynomial Kernel })\end{array}$ & $\mathrm{N} / \mathrm{A}$ & 91.37 \\
\cline { 2 - 4 } & $\begin{array}{c}\mathrm{R}=5, \mathrm{~m}=7 \\
\mathrm{RBF} \mathrm{kernel})\end{array}$ & $\mathrm{N} / \mathrm{A}$ & 82.10 \\
\hline $\begin{array}{c}\mathrm{k}-\mathrm{NN} \\
\text { Approach }\end{array}$ & $\mathrm{R}=5, \mathrm{~m}=7, \mathrm{k}=1$ & $\mathrm{~N} / \mathrm{A}$ & 85.50 \\
\cline { 2 - 4 } AdaBoost & $\mathrm{R}=5, \mathrm{~m}=7, \mathrm{k}=3$ & $\mathrm{~N} / \mathrm{A}$ & 87.73 \\
\hline PART & $\mathrm{R}=5, \mathrm{~m}=7$ & $\mathrm{~N} / \mathrm{A}$ & 82.17 \\
\hline J48 & $\mathrm{R}=5, \mathrm{~m}=7$ & $\mathrm{~N} / \mathrm{A}$ & 81.77 \\
\hline $\begin{array}{c}\text { Random } \\
\text { Tree }\end{array}$ & $\mathrm{R}=5, \mathrm{~m}=7$ & 80.83 \\
\hline
\end{tabular}

proposed classifier, the identification performance of the proposed classifier is compared with that of the already studied classifiers such as Bayesian Networks, SVM, kNearest Neighbors Approach, AdaBoost, PART, J48, and Random Tree. Weka was used to obtain identification performances of these already studied classifiers. Weka is the well-known machine learning tool which is a collection of machine learning algorithms and can be downloaded at the website www.cs.waikato.ac.nz. In this experiment, we use 10 -fold cross validation to evaluate the identification performance. Table 6 shows the comparison between the proposed classifier with the other well-known classification algorithms which are available in the Weka machine learning package. From the experimental results described in Table 6 , the proposed identification system is said to be superior to the other machine learning algorithms in terms of the prediction ability.

\section{Conclusion}

In this paper, we proposed the new identification system for black plastic based on a kind of intelligent system and Raman spectroscopy equipment. Near infrared radiation spectroscopy is usually used to extract spectrum of plastic material. However, when dealing with black plastic, near infrared radiation spectroscopy cannot be used. In order to extract the raw spectrum from black plastic, we use Rama spectroscopy. In addition, to improve the classification ability of the fRBFNNs, we apply a supervised clustering algorithm not an unsupervised learning method to determine the parameters of the hidden layers with aids of the auxiliary information. This auxiliary information involves the information which can be extracted over output space. From the experimental results, we can say that the auxiliary information can help improve the classification ability of RBF neural networks.

\section{Acknowledgements}

This study was supported by Basic Science Research Program through the National Research Foundation of Korea (NRF) funded by the Ministry of Science, ICT \& Future Planning [grant number NRF-2015R1A2A1A15055365] and also by the GRRC program of Gyeonggi province [grant number GRRC Suwon 2016-B2, Centre for U-city Security \& Surveillance Technology]

\section{References}

[1] Scott D. M., "A two color near-infrared sensor for sorting recycled plastic waste," Measurement Science and Technology, vol. 6, pp. 156-159, 1995.

[2] Edward, J. and Sommer J. R., "Method and Apparatus for Near Infrared Sorting of Recycled Plastic Waste," Patent in United States, Pub. No.: US 2001/0045518 A1, 2001.

[3] Sorely J. Cocjrane and Jordana Blacksberg, "A Fast Classification Scheme in Raman Spectroscopy for the identification of Mineral Mixtures Using a Large Database with Correlated Predictors," IEEE Trans. On Geoscience and Remote Sensing, vol. 53, No. 8, pp. 4259-4274, Aug. 2015.

[4] E. Smith and G. Dent, "Modern Raman SpectroscopyA Practical Approach," Hoboken, NJ, USA: Wiley, 2005.

[5] L. Zhang, K. Li, H. He, and G. W. Irwin, "A New Discrete-Continuous Algorithm for Radial Basis Function Networks Construction," IEEE Trans. On Neural Networks and Learning Systems, vol. 24, no. 11, pp. 1785-1798, 2013.

[6] W. Pedrycz, R. Al-Hmouz, and A. S. Balamash, "Hierarchical Granular Clustering: An Emergence of Information Granules of Higher Type and Higher Order," IEEE Trans. On Fuzzy Systems, vol. 23, no. 6, pp. 2270-2283, 2015.

[7] A. Gacek and W. Pedrycz, "Clustering Granular Data and Their Characterization with Information Granules of Higher Type," IEEE Trans. On Fuzzy Systems, vol. 23, no. 4, pp. 850-860, 2015.

[8] X. Q. Tang and P. Zhu, "Hierarchical Clustering Problems and Analysis of Fuzzy Proximity Relation on Granular Space," IEEE Trans. On Fuzzy Systems, vol. 21, no. 5, pp. 814-824, 2013.

[9] W. Pedrycz, "Conditional fuzzy C-Means," Pattern Recognition Letters, vol.17, no,6, pp.625-632, 1996.

[10] M.J. Er, S.Q. Wu, J.W. Lu, and H.L. Toh, "Face recognition with radical basis function (RBF) neural networks," IEEE Transactions on Neural Networks, vol. 13, no. 5, pp. 697-710, 2002.

[11] X.Y. Jing, Y.F. Yao, D. Zhang, J.Y. Yang, and M. Li, "Face and palm print pixel level fusion and Kernel 
DCV-RBF classifier for small sample biometric recognition," Pattern Recognition, vol.40, pp.3209 3224, 2007.

[12] S.-B. Roh, S.-K Oh, and W. Z. Choi, "Design of fuzzy radial basis function neural networks classifier based on conditional fuzzy C-means clustering algorithm," in conference of ICMIT 2015, pp.67 -70, 2015.

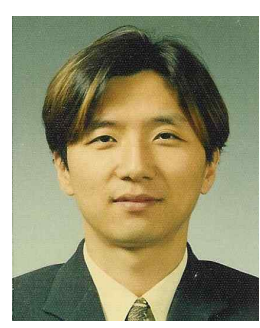

Seok-Beom Roh received the B.Sc., M.Sc., and Ph.D. degrees in control and instrumentation engineering from Wonkwang University, Korea, in 1994, 1996, and 2006 respectively. $\mathrm{He}$ is currently a Research Professor in the University of Suwon. His research interests include fuzzy set, neural networks, evolutionary algorithms, computational intelligence and statistical learning.

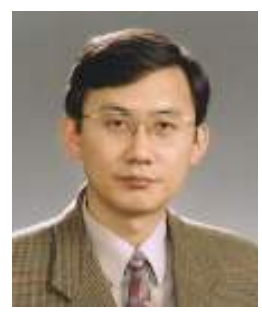

Sung-Kwun Oh He received the B.Sc., M.Sc., and Ph.D. degrees in electrical engineering from Yonsei University, Seoul, Korea, in 1981, 1983, and 1993, respectively. During 1983-1989, he was a Senior Researcher of R\&D Lab. of Lucky-Goldstar Industrial Systems Co., Ltd. From 1996 to 1997, he was a Postdoctoral Fellow with the Department of Electrical and Computer Engineering, University of Manitoba, Winnipeg, MB, Canada. He is currently a Professor with the Department of Electrical Engineering, The University of Suwon, Suwon, South Korea. His research interests include fuzzy system, fuzzy-neural networks, automation systems, advanced computational intelligence, and intelligent control. He currently serves as an Associate Editor of the KIEE Transactions on Systems and Control, International Journal of Fuzzy Logic and Intelligent Systems of the KFIS, and Information Sciences. 